\title{
An efficient broadcasting routing protocol WAODV in mobile ad hoc networks
}

\author{
M. Chekhar', K. Zine-Dine' ${ }^{2}$, M. Bakhouya ${ }^{3}$, A. Aaroud ${ }^{4}$, J. Gaber ${ }^{5}$ \\ ${ }^{1,4}$ LAROSERI Lab., Faculty of Sciences, Chouaib Doukkali University, El Jadida, Morocco \\ ${ }^{2}$ Mohammed V University in Rabat, Faculty of Sciences, Rabat, Morocco \\ ${ }^{3}$ International University of Rabat, Morocco \\ ${ }^{5}$ Université de Technologie Belfort-Montbeliard, Belfort, France
}

\begin{tabular}{l}
\hline \hline Article Info \\
\hline Article history: \\
Received Apr 9, 2019 \\
Revised May 28, 2021 \\
Accepted Jun 11, 2021 \\
\hline
\end{tabular}

Keywords:

Adaptive approaches

AODV

Broadcasting schemes

MANETS

Routing discovery

\begin{abstract}
Information broadcasting in wireless network is a necessary building block for cooperative operations. However, the broadcasting causes increases the routing overhead. This paper brings together an array of tools of our adaptive protocol for information broadcasting in MANETs. The proposed protocol in this paper named WAODV (WAIT-AODV). This new adaptive routing discovery protocol for MANETs, lets in nodes to pick out a fantastic motion: both to retransmit receiving request route request (RREQ) messages, to discard, or to wait earlier than making any decision, which dynamically confgures the routing discovery feature to decide a gorgeous motion through the usage of neighbors' knowledge. Simulations have been conducted to show the effectiveness of the using of techniques adaptive protocol for information broadcasting RREQ packet when integrated into ad hoc ondemand distance vector (AODV) routing protocols for MANET (which is based on simple flooding).
\end{abstract}

This is an open access article under the CC BY-SA license.

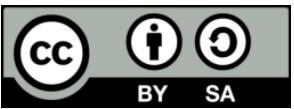

\section{Corresponding Author:}

Mohammed Chekhar

LAROSERI Lab., Faculty of Sciences

ChouaibDoukkali University

Route Ben Maachou, BP 20, Av des Facultes, El Jadida, Morocco

Email: chekhar.m@ucd.ac.ma

\section{INTRODUCTION}

Wireless communications have a crucial role to play in computer networks. They offer open solutions to provide mobility and essential services where infrastructure installation is not possible. Wireless networks are increasingly an emerging technology that allows users to access electronic information and services regardless of their geographic location. The success of this type of network in recent years has been driven by strong interest from individuals, businesses and industry. Indeed, this type of network is perceived as a new alternative to traditional wired networks [1].

In ad hoc on-demand distance vector (AODV), on-demand routing protocols [2], [3] protocol, each community node participates in the process as a router where the route request (RREQ) request messages are despatched from supply nodes, relayed through numerous intermediate nodes before attaining destination nodes. This operation leads to the hassle of broadcast storm [4]. Several routing protocols have been proposed for mobile ad hoc networks [5], [6], these routing protocols can be classified according to how routes are created and maintained during routing according to several criteria. Indeed, each node in the network must participate in the routing of packets through the network, thus playing the role of a router and a terminal at the same time. For this, a distributed routing protocol is required. However, such a network is 
subject to a number of constraints that make such a deployment very complex. These include: Radio medium constraints, the highly dynamic nature, and the lack of a centralized administration. For some applications such as multimedia or real-time applications the best effort service is not at all sufficient. Such applications require guarantees in terms of certain quality of service criteria (minimum bandwidth, and maximum delay not to be exceeded). Indeed, it seems important to adapt MANETs to support a certain level of QoS in order to deploy demanding applications.In this mode of communication, each mobile node of the network has the possibility to communicate directly with all its neighbors, i.e. all the nodes capable of receiving the signal sent and understanding it. Each node can move or disconnect from the network at any time. There is no infrastructure. We then speak of ad hoc networks or mobile ad hoc network (MANET).

As an extension to the work the authors proposed the adaptive broadcasting schemes in [7]-[9] to deal with these problems with recognize to packet delivery ratio (PDR) [10], normalized routing load, and average of end-to-end delay. In a latest work, in [11]-[14] the authors include more than a few mobility parameters to finds an most advantageous route discovery with minimal transmit energy. Simulations have been carried out and results exhibit that the proposed scheme has giant PDR, decrease in average end-to-end delay [15] and normalized routing load is maintained, in contrast to the AODV protocol (which is primarily based completely on convenient flooding) as well. The the relaxation of this paper is structured as follows: section 2 evaluations the simple AODV protocol mannequin proposed in literature for wireless ad hoc networks. In section 3, we current the proposed broadcasting scheme WAODV. Section 4 affords the simulation parameters, average overall performance metrics and offered results. Conclusions and future work are brought in section 5 .

\section{THE BASIC AODV PROTOCOL}

\subsection{Description of the AODV protocol}

The routing protocol AODV is a reactive routing protocol primarily based on the principle of distance vectors, capable of both unicast and multicast routing [16]. It essentially represents an improvement of the proactive DSDV algorithm [17]. This protocol uses both mechanisms "Route discovery" and "Route maintenance", it builds the routes by using of a query cycle "Route Request/Route Reply" of node by node. AODV use the principle of sequence number to maintain the consistency of recent routing information. In mobile ad hoc mobile networks, the routes frequently change because of the mobility of nodes. Hence, the route maintained by certain nodes become invalid. To use the fresh routes, the nodes use the sequence numbers. A node updates them whenever new information comes from a route request (RREQ), route replie (RREP), and route error (RERR) message, it increments its own sequence number in the following circumstances: i) It is itself the destination node and offers a new route to reach it; ii) It receives an AODV message (RREQ, RREP, and RERR) containing new information on the sequence number of a destination node; iii) The path to a destination is not valid. AODV continues the paths in a distributed manner by maintaining a routing table at every transit node embedded in the searched path.

\subsection{Route request mechanism (RREQ)}

AODV uses the principles of sequence numbers to maintain the consistency of routing information. Because of the mobility of nodes in ad hoc networks, routes change frequently, so that routes maintained by some nodes become invalid. Sequence numbers allow the use of the newest or freshest routes. AODV uses a route request to create a path to a certain destination.

However, AODV maintains paths in a distributed fashion by keeping a routing table at each transit node belonging to the path being searched. A node forward a route request discovery packet RREQ in case it wishes to recognize a route to a sure destination and such a route is now not accessible as shown in Figure 1. This can occure if the destination is no longer known beforehand, or if the current course to the destination has expired its lifetime or has become faulty (i.e. the metric related with it is infinite). The field, destination sequence range of the RREQ packet, consists of the ultimate acknowledged price of the sequence variety associated with the destination node.

After the broadcast of the route request discovery packet RREQ, the source is ready for the route response packet (RREP). If the latter is no longer obtained during a sure period, the supply can rebroadcast a new RREQ request. This statistics used to construct the reverse path as shown in Figure 2, which will be traversed through the unicast route response packet.

Since the route reply RREP packet will be sent to the source, the nodes belonging to the return direction will regulate their routing tables following the direction contained in the response packet. In order to limit the cost in the network, AODV proposes to extend the search progressively. Initially, the query is broadcastto a limited number of hops. If the source receives no response after a specified timeout, it will retransmit another search message by increasing the maximum number of hops. In case of no answer, this 
procedure is repeated a maximum number of times before declaring this destination unreachable. With each new broadcast, the Broadcast ID field of the RREQ packet is incremented to identify a particular route request associated with a source address.

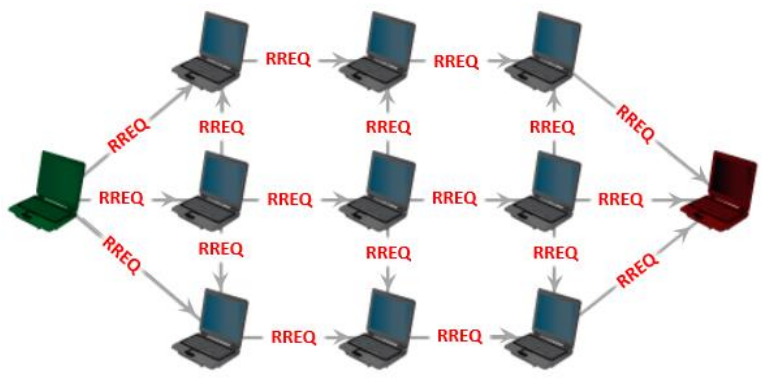

Figure 1. Propagation of route request (RREQ) packet

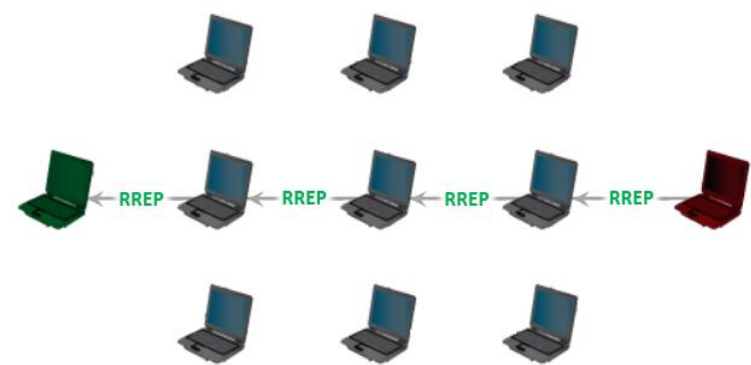

Figure 2. Path taken by the route reply (RREP) packet

If the request RREQ packet is rebroadcast a number of times (RREQ_RETRIES) without receiving a response, an error message is issued to the application. The destination returns a message RREP, this message can be routed to the source. Each traversed node will increment the number of jumps. In addition, add an entry to his table for the destination. A node located between the source and the destination can also give an adequate response. In this case, obtaining bidirectional routes is nevertheless possible thanks to the "Freeous RREP" flag. The intermediate node will then send a RREP to the destination. The nodes between the intermediate node and the destination will therefore add to their table an entry to the source of the RREQ. This arrangement will allow the destination to send packets directly to the source without having to search for a route. This is useful when establishing TCP communications for sending the first ACK [18].

\section{WAODV SCHEME}

Since the WAODV [19] protocol is inspired by the AODV protocol, it retains most of these operating mechanisms with modifications during the broadcast of the route discovery request. In addition, to facilitate the admission control performed during the RREQ broadcast, a dynamic approach will be used to estimate the replay of the RREQ request. Using the periodic broadcast of RREQ messages performed by the WAODV protocol, each node then infers its own choice of replay. Another RAD value will be calculated periodically for each node. This will be used when receiving an RREQ to calculate the likely time spent in the queue of the node considered the RREQ for use in admission control performed by the WAODV protocol.

If a source node wants to communicate with another node that does not have a valid route in its routing table for that destination, a route discovery procedure is initiated. When an intermediate node receives an RREQ, it first checks if there is a valid route in the routing table, if there is a route it sends an RREP back to the source, if not, before retransmitting the message, it performs an admission check to test the delay. This consists in comparing the value of the RAD field of the RREQ request with the calculated delay.

The protocol proposed in this study, called WAODV (WAIT-AODV) [19], agrees to contract to choose an appropriate first-order action, i.e. retransmit, ignore a received RREQ packet, or wait before any selection is made (i.e., now not too a great deal statistics handy so a long way to make proper decision). The proposed protocol relies entirely on gaining knowledge of the model that collects neighbor statistics such as a variety of RREQ packets received and their arrival time $t(s)$ to prevent some nodes from rebroadcasting using appropriate action selection (i.e., rebroadcast, discard).

Unlike fully based AODV or modified AODV protocols proposed from the literature that choose between two movements [20], [21], the proposed new protocol adds every other procedure, called waiting, in addition to ignoring and rebroadcasting. In other words, when a node receives a broadcast RREQ packet, it takes advantage of the neighbors' understanding to determine the appropriate action with the help of another addition (wait procedure). A decision to rebroadcast or discard the request message.

For example, a rebroadcast should be specified when neighboring nodes do not receive any request, while a wait action is chosen when the node cannot make any choice due to the fact of lack of information. Exclusion traffic must be determined when a node receives the same packet at one time. It is the capacity of neighboring nodes that have already received the matched request packet, that is, more copies that the node receives. 
The precept of this mannequin is described as follows. The node received a RREQ route request discovery packet for the first time at instance $t_{a}$, the host starts a counter S. A random evaluation delay RAD $(\tau)$ to also be generated for the RREQ request broadcast. During $\tau$, if RREQ was heard again at instance $t_{b}$, and Update table $T\left[\Delta t_{i}\right]$; by recording $\Delta t_{i}=\left(t_{b}-t_{a}\right)$, and increases counter $\mathrm{S}$, and when $\tau$ has expired, if RREQ received only once, Rediffuse RREQ request packet and exit the procedure. Otherwise the system takes the $\Delta t_{i}$ values stored in the table and compares them with $\tau$ : when $\Delta t_{i}$ is less than one third of $\tau$, then decreases the counter $\mathrm{S}$, if the latter is less than two thirds of $\tau$ then the system increases counter $\mathrm{S}$. finally if the counter positive, then Rediffuse the RREQ request packet and exit the procedure, Otherwise the RREQ will be dropped directly by this intermediate node and exit the procedure. The rebroadcasting selection algorithm is as follows: (The algorithm is proven beneath in detail.)

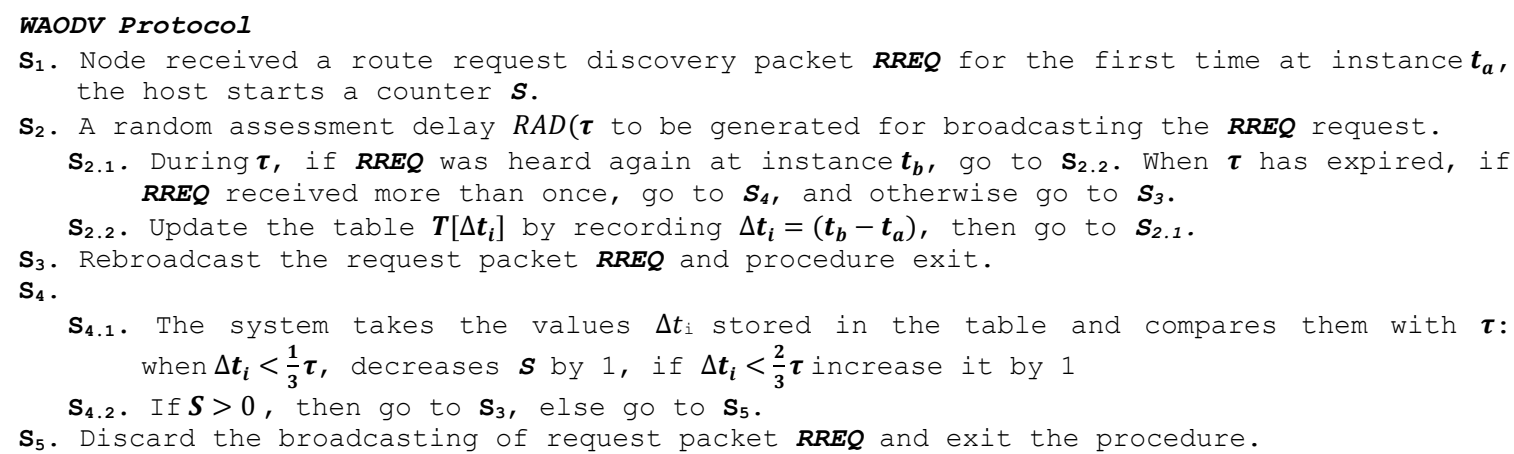

\section{PERFORMANCES EVALUATION}

This section describes the simulation parameters, common overall performance metrics used in our contrast and finally simulation consequences the use of network simulator NS2. We in general focus on evaluating and evaluation the proposed model with the fundamental protocol AODV. Nonetheless, simulations parameters and performance metrics are first to show the testbed environment. Simulation consequences are then to study the influence of network density and traffic load on considered performance metrics.

\subsection{Simulation parameters}

Since the goal of our simulations is to analyze the properties of the AODV protocol extension, we chose that the traffic sources be constant bit rate (CBR). Traffic between nodes is generated by initializing CBR traffic connections that start at fixed times via the simulation script as shown in Table 1. The size of the data packets is 1000 bytes. We did not employ TCP traffic sources for example because TCP offers a statecompliant load on the network, i.e., TCP traffic changes the times at which it sends packets based on its perception of the network's ability to deliver these packets [17].

Table 1. Simulation parameters

\begin{tabular}{cc}
\hline Simulation Parameters & Value(s) \\
\hline Simulator & NS-2 version $(2.34)$ \\
Network Range & $1000 \mathrm{~m} \times 1000 \mathrm{~m}$ \\
Transmission Range & $250 \mathrm{~m}$ \\
Number of nodes & $50,100,150,200,250,300$ \\
Route Bandwidth & $2 \mathrm{Mbps}$ \\
Connection Type & CBR $-10,20,30,40,50 \mathrm{Connection}$ \\
Nodes Speed & Min $1(\mathrm{~m} / \mathrm{s})$, Max $5(\mathrm{~m} / \mathrm{s})$ \\
Message size & 1000 bytes \\
Packet Rate & 4 Packets $/ \mathrm{Sec}$ \\
Number of trial & 30 \\
Interface Queue length & 50 \\
\hline
\end{tabular}

In this study, we used such a community configuration to limit the possibility of a partitioning of network taking place for the duration of the simulation time. In addition, these values have been chosen due to the fact they are on computing and time assets for running the most scenarios. A node mobility situation used to be generated the use of random-waypoint mannequin [22]. Furthermore, with the aid of varying nodes' velocity from minimal speed its price is set to one meter per second up to maximum speed cost equal to five meters per second. In the beginning of the simulation the nodes stay motionless in the course of a pause 
time and each node chooses a random destination, then begins transferring towards it with velocity is various from 1 to $5 \mathrm{~m} / \mathrm{s}$. Additionally, this cycle repeats until the simulation terminates for investigating the conduct of the proposed protocol when various the site visitors load by using it constant bit rate (CBR) [23]-[25]. Table 1 lists the parameters used in our simulations.

In the rest of this section, results related to our investigation to show the effect of nodes density within the range from 50 to 300 nodes according traffic load of network varying from 10 to 50 . However, we measured and compared the performance of the WAODV scheme, under different network densities and traffic load of network, against the basic AODV protocol. The performance metrics evaluated are as follows: Packet delivery ratio (PDR), normalized routing load (NRL), and average of end-to-end delay. This protocol could give significant results without basic protocol.

\subsection{Simulation results and discussion}

The WAODV scheme was implemented and integrated into the network simulator-2 (NS-2) version 2.34 [26] and compared it to fundamental AODV protocol. We frequently investigated the traffic load of network's within the CBR connection as 10, and by varying number of nodes from 50 to 150 nodes. Where a dynamic topology where nodes speed is fixed to 1 , and $5 \mathrm{~m} / \mathrm{s}$. We performed thirty simulation trials for each scenario and computed the average number of NRL, PDR, and average end-to-end delay, and obtained with each scheme. The simulation model consists of two sets of scenario files: topology scenario and traffic generation files. Figure 3 presents results that illustrate the effect of network's density on PDR for the all schemes. We can see that the PDR decreases barely with the make bigger in network's density for all schemes.

The WAODV protocol has considerable PDR as in contrast to the simple AODV and this underneath unique network's density. More precisely, outcomes verify that in WAODV, data obtained by using neighbors was once really used to enhance the getting to know process. WAODV protocol has greater PDR, that implying for this reason some nodes do now not want to rebroadcast the message, i.e., it inhibit extra nodes from rebroadcasting in contrast to the simple AODV protocol.

Figure 4 shows the comparison of WAODV protocol against of the basic AODV. We can see that all the protocols showing a force increase in terms of NRL with a different density of networks. In AODV routing protocol, NRL is increase with increase in number of node in network. The NRL of our AODV improvement is the highest compared to the AODV base protocol with the increase in node density. AODV demonstrates significantly lower routing load and fairly stable as compare to WAODV with an increasing number of sources.

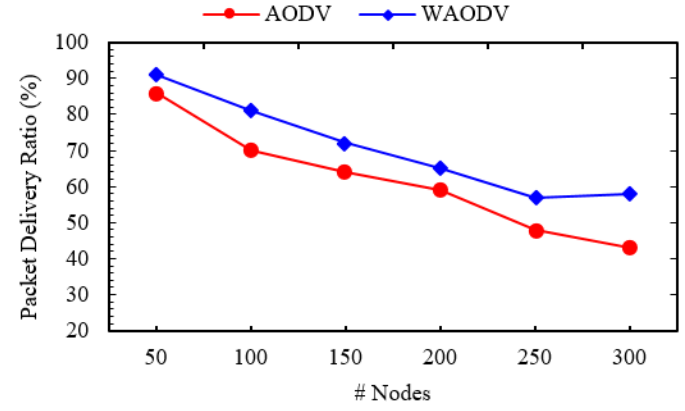

Figure 3. Packet delivery ratio vs. number of nodes

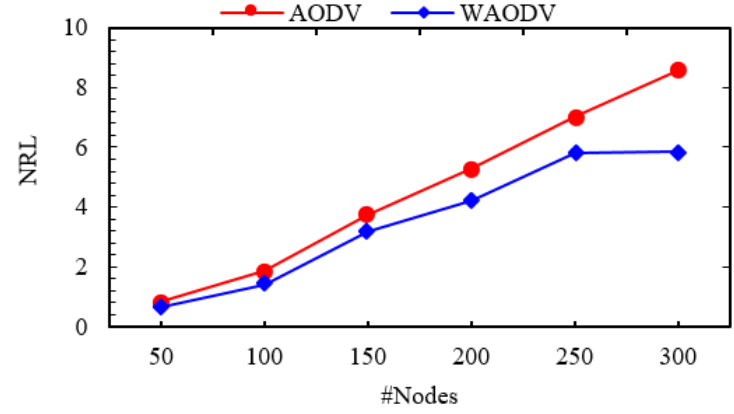

Figure 4. Normalized routing load vs. number of nodes

Figure 5 depicts average end-to-end delay values according of network's density. As proven in this figure, the quicker the node density is. The greater end-to-end delay is incurred. The AODV protocol has the best delay. This is due to the reality that, in flooding protocol, the numbers of retransmissions is very excessive and messages are queued for lengthy time. In WAODV protocol, the common extend is low in contrast to AODV protocol due to the fact the wide variety of retransmissions is additionally low and then the queue time at every node is short. Moreover, this discern indicates that AODV simple has greater and unstable end-to-end delay cost as a end result of the greater range of redundant rebroadcasts of RREQ packets; this can be defined through with contentions and collisions in which many RREQ packets fail to attain the destination, ensuing in growing delay.

Figure 6 depicts Packet delivery ratio according of traffic load of network for WAODV and basic AODV. This discern offers that our proposed protocol has a greater cost of PDR in contrast with AODV 
basic. However, Packet delivery ratio will increase as the range of connections increases. This potential that there are extra connections to join two nodes and facilitate the transmission in every area. Thus, there is a increased risk that the broadcast will be correctly resubmitted, increasing the delivery ratio.

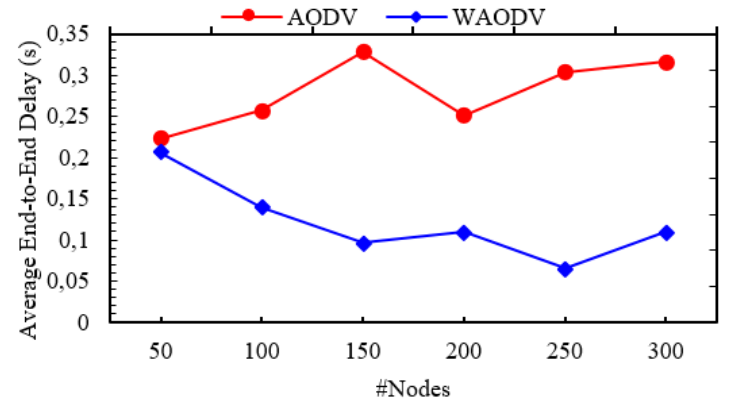

Figure 5. Average end-to-end delay vs. number of nodes

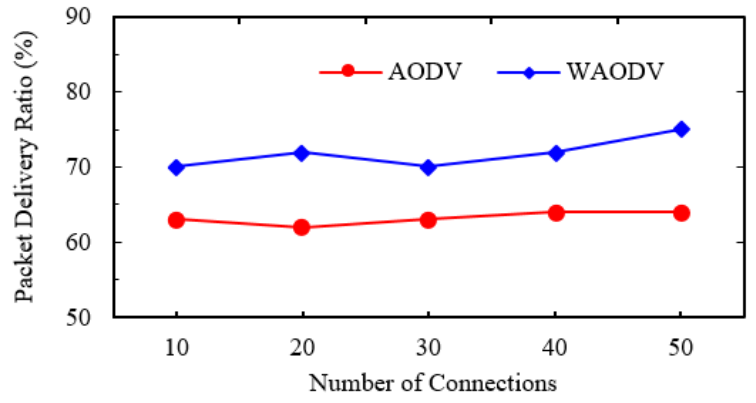

Figure 6. Packet delivery ratio vs. traffic load of network

Figure 7 depicts NRL values for differenttraffic load of network of network size 150 nodes. Figure 6 shows the NRL comparison of WAODV with the basic AODV protocols. We can see that the NRL increases as traffic load of network increases. This is due to the increase in packets retransmissions. We can see also that our proposed approach exhibits the lowest traffic load percentage against other approachs. According to results shown in Figures 6-8, WAODV has significant NRL, good PDR, and lower delay compared to the AODV basic protocol. It is the most efficient proposal with respect to considered metrics in terms of both network density and traffic load of network.

The Figure 8 shows the average delay results that all packets experience from the moment they are sent by the source nodes until they reached all the nodes. This figure depicts average end-to-end delay at various traffic load of network. WAODV is shown as the most efficient protocol because packets traverse fewer hops, because of its adaptive nature according to the traffic load.

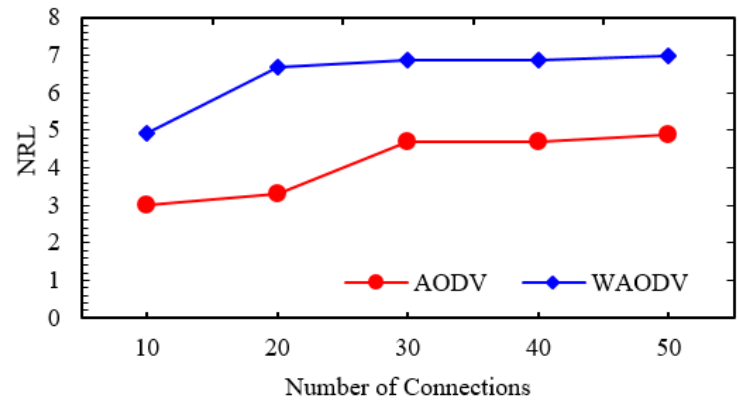

Figure 7. Normalized routing load vs. traffic load of network

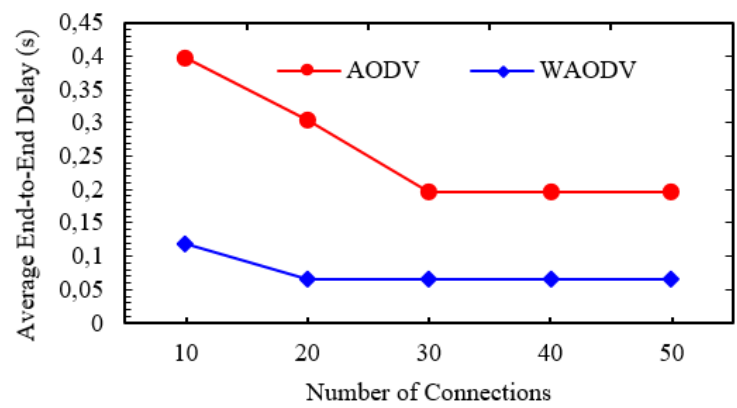

Figure 8. Average delay vs. traffic load of network

\section{CONCLUSION}

This paper describes the specifications of a solution that extends the AODV protocol to guarantee RREQ packet replay. It also details the working principle of the new protocol and the method used for the estimation of the RREQ packet replay. This article was dedicated to the design of WAODV. In it, we have discussed the main phases that show the relationships between the different classes of the protocol. We presented a simulation to analyze the properties of the WAODV protocol extension with basic AODV using the NS2 network simulator. The simulation results were plotted on graphs and interpreted. These simulations led us to know well how the WAODV protocol operates in the face of network density and traffic load as well as to validate the variation of the connection acceptance rate in the presence of admission control based on bandwidth availability in the routing nodes. 


\section{REFERENCES}

[1] S. Corson and J. Macker, "Mobile ad hoc networking (MANET): Routing protocol performance issues and evaluation considerations," RFC Editor, United States, 1999, doi: 10.17487/RFC2501.

[2] I. D. Chakeres and E. M. Belding-Royer, "AODV routing protocol implementation design," 24th International Conference on Distributed Computing Systems Workshops, 2004. Proceedings, 2004, pp. 693-703, doi: 10.1109/icdesw.2004.1284108.

[3] D. B. Johnson and D. A. Maltz, "DSR: The Dynamic Source Routing Protocol for Multi-Hop Wireless Ad Hoc Networks," Ad hoc networking, vol. 5, no. 1, pp. 139-172, 2001, doi: 10.1007/BF01193336.

[4] Y. C. Tseng, S. Y. Ni, Y. S. Chen, and J. P. Sheu, "The broadcast storm problem in a mobile ad hoc network," Wireless Networks, vol. 8, no. 2/3, pp. 153-167, 2002, doi: 10.1023/A:1013763825347.

[5] M. Chekhar, K. Zine-Dine, M. Bakhouya, and A. Aaroud, "A dynamic threshold-based probabilistic scheme for broadcasting in ad hoc networks," 2015 15th International Conference on Intelligent Systems Design and Applications (ISDA), 2016, pp. 511-516, doi: 10.1109/ISDA.2015.7489168.

[6] M. Bakhouya and J. Gaber, "Approaches for engineering adaptive systems in ubiquitous and pervasive environments," Journal of Reliable Intelligent Environments volume, vol. 1, pp. 75-86, 2015, doi: 10.1007/s40860015-0010-6.

[7] V. B. Kute and M. U. Kharat, "Analysis of quality of service for the AOMDV routing protocol," Engineering Technology and Applied Science Research, vol. 3, no. 1, pp. 359-362, 2012, doi: 10.48084/etasr.105.

[8] P. Gupta, P. Goel, P. Varshney, and N. Tyagi, "Reliability Factor Based AODV Protocol: Prevention of Black Hole Attack in MANET," Smart Innovations in Communication and Computational Sciences, vol. 851, pp. 271-179, 2019, doi: 10.1007/978-981-13-2414-7_26.

[9] T. Kabir, N. Nurain, and M. H. Kabir, "Pro-AODV (Proactive AODV): Simple modifications to AODV for proactively minimizing congestion in VANETs," 2015 International Conference on Networking Systems and Security (NSysS), 2015, pp. 1-6, doi: 10.1109/NSysS.2015.7043521.

[10] L. Liu, C. Chen, T. Qiu, M. Zhang, S. Li, and B. Zhou, "A data dissemination scheme based on clustering and probabilistic broadcasting in VANETs," Vehicular Communications, vol. 13, pp. 78-88, 2018, doi: 10.1016/j.vehcom.2018.05.002.

[11] M. Rath, B. Pati, and B. K. Pattanayak, "Design and development of secured framework for efficient routing in vehicular ad-hoc network," International Journal of Business Data Communications and Networking (IJBDCN), vol. 15, no. 2, pp. 55-72, 2019, doi: 10.4018/IJBDCN.2019070104.

[12] H. Alani, M. Abdelhaq, and R. Alsaqour, "Dynamic routing discovery scheme for high mobility in mobile ad hoc wireless networks," International Journal of Electrical and Computer Engineering (IJECE), vol. 10, no. 4, pp. 3702-3714, 2020, doi: 10.11591/ijece.v10i4.pp3702-3714.

[13] T. O. Fahad and A. A. Ali, "Compressed fuzzy logic based multi-criteria AODV routing in VANET environment," International Journal of Electrical and Computer Engineering (IJECE), vol. 9, no. 1, pp. 397-401, 2019, doi: 10.11591/ijece.v9i1.pp397-401.

[14] C. Wu, S. Ohzahata, and T. Kato, "Flexible, portable, and practicable solution for routing in VANETs: A fuzzy constraint Q-learning approach," IEEE Transactions on Vehicular Technology, vol. 62, no. 9, pp. 4251-4263, 2013, doi: 10.1109/TVT.2013.2273945.

[15] Q. Zhang and D. P. Agrawal, "Performance evaluation of leveled probabilistic broadcasting in MANETs and wireless sensor networks," Simulation, vol. 81, no. 8, pp. 533-546, 2005, doi: 10.1177/0037549705060234.

[16] T. K. Saini and S. C. Sharma, "Prominent unicast routing protocols for Mobile Ad hoc Networks: Criterion, classification, and key attributes," Ad Hoc Networks, vol. 89, pp. 58-77, 2019, doi: 10.1016/j.adhoc.2019.03.001.

[17] T. E. Ali, L. A. K.Al Dulaimi, and Y. E. Majeed, "Review and performance comparison of VANET protocols: AODV, DSR, OLSR, DYMO, DSDV \& ZRP," 2016 Al-Sadeq International Conference on Multidisciplinary in IT and Communication Science and Applications (AIC-MITCSA), 2016, pp. 1-6, doi: 10.1109/AICMITCSA.2016.7759934.

[18] A. L. Lugo and J.-P. Hubaux, "TraNS: realistic joint traffic and network simulator for VANETs," ACM SIGMOBILE Mobile Computing and Communications Review, vol. 12, no. 1, pp. 31-33, 2008, doi: 10.1145/1374512.1374522.

[19] M. Chekhar, K. Zine-Dine, M. Bakhouya, A. Aaroud, and D. El Ouadghiri, "An Efficient Broadcasting Scheme in Mobile Ad-Hoc Networks," Procedia Computer Science, vol. 98, pp. 117-124, 2016, doi: 10.1016/j.procs.2016.09.019.

[20] M. B. Yassein, S. F. Nimer, and A. Y. Al-Dubai, "A new dynamic counter-based broadcasting scheme for Mobile Ad hoc Networks," Simulation Modelling Practice and Theory, vol. 19, no. 1, pp. 553-563, 2011, doi: 10.1016/j.simpat.2010.08.011.

[21] S. S. Basurra, M. De Vos, J. Padget, Y. Ji, T. Lewis, and S. Armour, "Energy efficient zone based routing protocol for MANETs," Ad Hoc Networks, vol. 25, pp. 16-37, 2015, doi: 10.1016/j.adhoc.2014.09.010.

[22] W. Peng and X. C. Lu, "On the reduction of broadcast redundancy in mobile ad hoc networks," 2000 First Annual Workshop on Mobile and Ad Hoc Networking and Computing. MobiHOC (Cat. No. OOEX444), 2000, pp. 129-130, doi: 10.1109/MOBHOC.2000.869221.

[23] K. Xu, D. Tipper, Y. Qian, P. Krishnamurthy, and S. Tipmongkonsilp, "Time-varying performance analysis of multihop wireless networks with CBR traffic," IEEE Transactions on Vehicular Technology, vol. 63, no. 7, pp. 3397-3409, 2014, doi: 10.1109/TVT.2013.2297382. 
[24] M. Bakhouya, J. Gaber, and P. Lorenz, "An adaptive approach for information dissemination in Vehicular Ad hoc Networks," Journal of Network and Computer Applications, vol. 34, no. 6, pp. 1971-1978, 2011, doi: 10.1016/j.jnca.2011.06.010.

[25] IEEE, "Part 11: Wireless LAN Medium Access Control (MAC) and Physical Layer (PHY) Specifications," 2012

[26] "Network Simulator NS 2.34," [Online]. Available: http://www.isi.edu/nsnam

\section{BIOGRAPHIES OF AUTHORS}
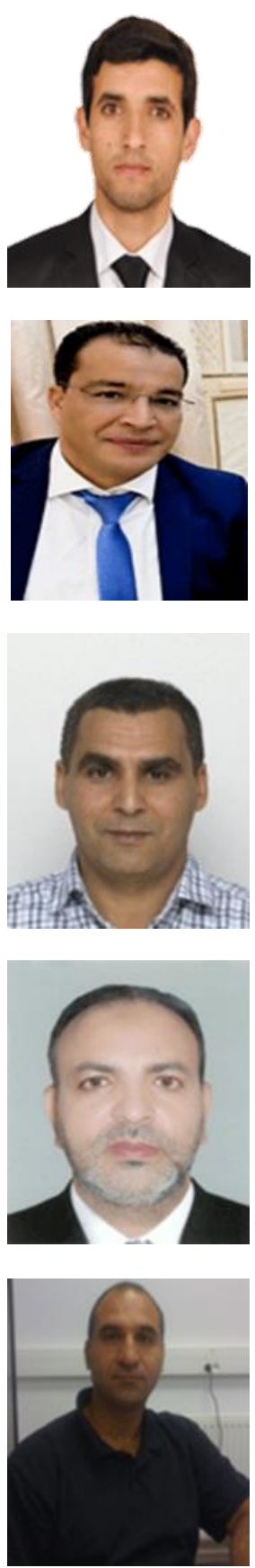

Mohammed Chekhar received his PhDat the Faculty of Sciences, University ChouaibDoukkali (UCD), Morocco, in 2019. He received his M.S. degree in Telecommunications \& Networking and B.S. degree in Physics from UCD in 2011 and 2009 respectively. His current research focuses on MANET communication protocols, wireless ad hoc Networks, and mobile computing.

Khalid Zine-Dine received his $\mathrm{PhD}$ degree from the Mohammed V University of Rabat, Morocco, in 2000. He spent four years in bank Information System as a network \&system security project manager. Currently, he is an Associate Professor at Faculty of Sciences Mohammed V University in Rabat, Morocco. His research interests are in the area of wireless ad hoc and sensor networks, Mobility, security, cloud computing and system \& network architectures and protocols. Recently, he has been interested in the field of MicroGrid and energy efficiency. Dr. Zine-Dine was a co-organizer and co-chair of conferences and is involved in many $\mathrm{PhD}$ Thesis and funded projects.

Mohamed Bakhouya is an associate professor at International University of Rabat. He obtained his HDR from UHA-France in 2013 and his PhD from UTBM-France in 2005. Bakhouya's research has been supported by many national and international grants. He is EiC of IJARAS journal and also serves as a guest editor of a number of international journals, ACM Trans. on Autonomous and Adaptive Systems, Product Development Journal, Concurrency and Computation: Practice and Experience, FGCS, and MICRO. His research interests include various aspects on the design, validation, and implementation of distributed and adaptive systems, architectures, and protocols. He is member of IEEE and ACM.

Abdessadek Aaroud is a Professor at ChouaibDoukkali University (Morocco) He received his $\mathrm{PhD}$ in 2004 from CaddiAyyad University in Marrakesh. His research interests iclude communication systems and Ad hoc network: theory and applications.

Jaafar Gaber received the PhD. degree in 1998 from University of Science and Technology of Lille (France) in Computer Science. He is currently an Associate Professor of Computational Sciences and Computer Engineering at the UBM. Prior to joining UBM, he was a research scientist at the Institute of Computational Sciences and Informatics (CSI) in George Mason University in Fairfax (Virginia, USA). Gaber's research has been supported by many European grants within EU FP6 and FP7 projects. His research interests include high-performance and reconfigurable computing, distributed data mining, Bio-computing, distributed algorithms and mobile computing. 